\title{
An Accretion-Disc Model for the Algol-Type Eclipsing Binary System AV Del
}

\author{
S. M. R. Ghoreyshi ${ }^{\mathrm{A}, \mathrm{C}}, J$. Ghanbari $^{\mathrm{A}, \mathrm{B}}$, and F. Salehi ${ }^{\mathrm{B}}$ \\ ${ }^{A}$ Department of Physics, School of Sciences, Ferdowsi University of Mashhad, Mashhad, Iran \\ B Department of Physics, Khayyam Institute of Higher Education, Mashhad, Iran \\ ${ }^{\mathrm{C}}$ Corresponding author. Email: smrgho@gmail.com
}

Received 2010 April 13, accepted 2010 September 14

\begin{abstract}
This study inspects the light and radial-velocity curves of the eclipsing binary AV Del. In comparison with other studies already done, the study shows that the absolute elements, fundamental orbital and physical parameters of the system can be determined using the Wilson-Devinney code. Using these parameters, the configuration of the system is presented. Then, an accretion disc model for the system is introduced by using the SHELLSPEC code. The results indicate that AV Del is a semi-detached system in which an optically thick accretion disc is surrounding the primary star. The outer radius of the disc is $8.0 R_{\odot}$, corresponding to a distance of $1.1 R_{\odot}$ from the surface of the secondary. Also, the temperature of the disc is calculated to be $T=5700 \mathrm{~K}$.
\end{abstract}

Keywords: variable stars — binaries — eclipsing binary — accretion disc

\section{Introduction}

AV Del $\quad\left(\alpha_{2000}=20^{h} 45^{m} 31^{s} .47, \quad \delta_{2000}=11^{\circ} 10^{\prime} 26^{\prime \prime} .4\right.$, $V=11.8)$ is a rather neglected variable star which was discovered by Hoffmeister (1935), and was given the original designation 184.1930. It is a suspected member of the cool Algols (Popper 1996) and was reported by the author as a double-lined system with a period of 3.85 days and a mean spectral type of G5, although other sources have classified it as F8 (e.g. Halbedel 1984). The rare class of cool Algol systems are distinguished from the classical Algol systems in that the mass-gaining component is a late-type star rather than a B- or A-type star. Mader et al. (2005) presented a new spectroscopic and $B V R I$ photometric observations of AV Del. They determined the radial velocities of both components using the two-dimensional cross-correlation technique TODCOR (Zucker \& Mazeh 1994). To present a detailed radialvelocity and light-curve analysis of the optical data, Mader et al. (2005) combined all light-curve data with the spectroscopic observations and analyzed them using the Wilson-Devinney (WD) code (Wilson \& Devinney 1971; Wilson 1979, 1990). They showed the system to be most likely semi-detached, with the less massive and cooler star filling its Roche lobe.

When a star (part of a binary star) in a circular orbit expands to fill and then overfill its Roche lobe, it will start to lose mass to its companion. Whether or not the mass transfer can proceed in a steady stable manner depends largely on the relative rates of change of stellar radius and Roche lobe radius, with respect to change in the loser's mass. Also, it depends on a steady overfilling of the Roche lobe which can be influenced by, for example, the magnetic field (magnetic activity cycle) of the cool component.

Matter that leaves the surface of one component of a binary star can be partly or wholly accreted by the companion. The matter can directly strike the gainer, or it can be stored in an accretion disc around it, and a part of the matter may leave the system. The question of which possibility is realized mainly depends on the orbital period and the separation of the components of the system. In fact, the disc may be caused by a binary star interaction. The study of accretion discs is one of the most active areas in stellar astrophysics.

Many objects that involve accretion discs are accompanied by bipolar jets that appear to emerge from the central region normal to the disc. Also, in the place where the gas stream from the secondary strikes the edge of the disc, a bright spot may be formed. Moreover, a thin shell of gas may surround the primary star that usually expands because of the stellar wind of the primary.

All of these effects can play an important role in the evolution of binary systems. Therefore, studying the existence and structure of these effects can give us insights into interacting binary systems.

As mentioned earlier, the Algol-type binaries are interacting systems consisting of a hot, usually more massive, main-sequence star and a cool giant or subgiant that fills its Roche lobe. The secondary is losing mass to its companion, and we observe the circumstellar material in the form of a gas stream, a jet, a shell, an accretion annulus, or an accretion disc. The most recent paper that summarizes our current knowledge of the Algols is the work by Richards \& Albright (1999). 
Table 1. The limb-darkening coefficients for AV Del

\begin{tabular}{lccccccrr}
\hline Parameters & $x_{1}(\mathrm{bol})$ & $x_{2}(\mathrm{bol})$ & $y_{1}(\mathrm{bol})$ & $y_{2}(\mathrm{bol})$ & $x_{1}$ & $y_{1}$ & $x_{2}$ & $y_{2}$ \\
\hline Filter $B$ & 0.639 & 0.632 & 0.225 & 0.150 & 0.739 & 0.253 & 0.813 & -0.038 \\
Filter $V$ & 0.639 & 0.632 & 0.225 & 0.150 & 0.823 & 0.165 & 0.842 & -0.232 \\
Filter $R$ & 0.639 & 0.632 & 0.225 & 0.150 & 0.647 & 0.272 & 0.750 & 0.092 \\
Filter $I$ & 0.639 & 0.632 & 0.225 & 0.150 & 0.554 & 0.265 & 0.652 & 0.150 \\
\hline
\end{tabular}

As we see in section 2, there is evidence for a mass transfer in AV Del. This paper examines the existence of the mass transfer and the nature of the system. First, it presents the analysis of the system using the latest version (Van Hamme \& Wilson 2007) of the wD code in section 3 and shows a new analysis of light and radial-velocity curves, then determines the physical and absolute parameters of the system. The study is replicated without following that of Mader et al. (2005) because it uses the latest version of the WD code and should obtain more parameters of the system with more accuracy than those found by Mader et al. (2005).

Although the wD code is very useful in the study of the physical parameters of the primary and secondary stars of binary systems, it is not suitable for exploring objects like discs, jets, spots, streams and shells. However, it is likely that these objects play an important role in Algol-type binaries. Although the wD code has an adjustable parameter $l_{3}$ (third light), its equations are not complete for a detailed study of the objects are listed above. Therefore, the SHELLSPEC code (Budaj \& Richards 2004) is used, which is a strong program for the analysis of those stars which have accretion discs and circumstellar material. Analysis of the system using the SHELLSPEC code is explained in section 4 .

\section{Assumptions}

This paper considered the $B, V, R$, and $I$ filter observations of Mader et al. (2005). The latest version of the wD code was applied to solve the light and radial-velocity curves. After obtaining the absolute parameters of the components, the SHELLSPEC code was used to derive the parameters of the disc. Throughout this paper, the subscripts 1 and 2 refer to the primary (hotter) and the secondary (cooler) components, respectively.

In the process of using the WD code, for both components of the system, bolometric linear, logarithmic and square-root laws for the limb darkening were used and the optimal results were obtained for the bolometric logarithmic limb-darkening law of Klinglesmith \& Sobieski (1970) as follows:

$$
I=I_{\circ}(1-x+x \cos \theta-y \cos \theta \ln (\cos \theta)),
$$

where the limb-darkening coefficients $x$ and $y$ for both components were fixed to their theoretical values, interpolated using Van Hamme's (1993) formula, which are depicted in Table 1.
The gravity-darkening exponent from Lucy (1967) and the bolometric albedos from Rucinski (2001) were chosen for convective envelopes ( $g=0.32, A=0.5)$, which are in line with the final surface temperatures. In order to reduce the number of free parameters, these parameters were kept constant during all iterations. It was assumed that the binary system has zero orbital eccentricity $(e=0.0)$ and its rotational and orbital spins are synchronous $\left(F_{1}=\right.$ $F_{2}=1.0$ ). Also, the black-body radiation models and the stellar atmosphere formulation for local emission on the primary and secondary stars were used, and the optimal results were obtained for the black-body radiation models. Initially, solutions were obtained assuming $l_{3} \neq$ 0.0 , but this assumption never resulted in satisfactory fits to the observations.

Mader et al. (2005) calculated a linear ephemeris:

$$
\begin{aligned}
\operatorname{Min} . I(H J D)= & 2,450,714.34779(39) \\
& +3.8534528(35) E,
\end{aligned}
$$

where $E$ is the number of cycles counted from the epoch of reference and the uncertainties are given in parentheses in units of the last significant digits. A period study of AV Del was carried out by Qian (2002) on the basis of fewer eclipses. A short period increase of about $3.15 \pm 0.19 \times$

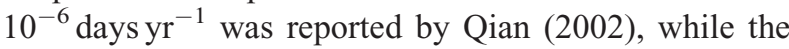
other quadratic fit derived by Mader et al. (2005) gives:

$$
\begin{aligned}
\operatorname{Min} . I(H J D)= & 2,450,714.34771(34) \\
& +3.8534620(42) E \\
& +0.50(16) \times 10^{-8} E^{2},
\end{aligned}
$$

which corresponds to a period change of $0.95 \pm 0.30 \times$ $10^{-6}$ days $\mathrm{yr}^{-1}$. These period changes may be related to the mass transfer presumably taking place in the system (Mader et al. 2005). The main purpose of this paper is to check the possibility of the presence of an accretion disc in AV Del by applying model analysis to the existing light curves.

\section{WD Solutions}

The photometric data reported by Mader et al. (2005) was used for analysis by the wD code. Values were specified for the inclination angle $(i)$, the mass ratio $\left(q \equiv M_{2} / M_{1}\right)$, the modified gravitational potentials ( $\Omega$, in the wD usage), the relative monochromatic luminosity of the primary $\left(L_{1}\right)$ and the mean temperature of the secondary $\left(T_{2}\right)$. 


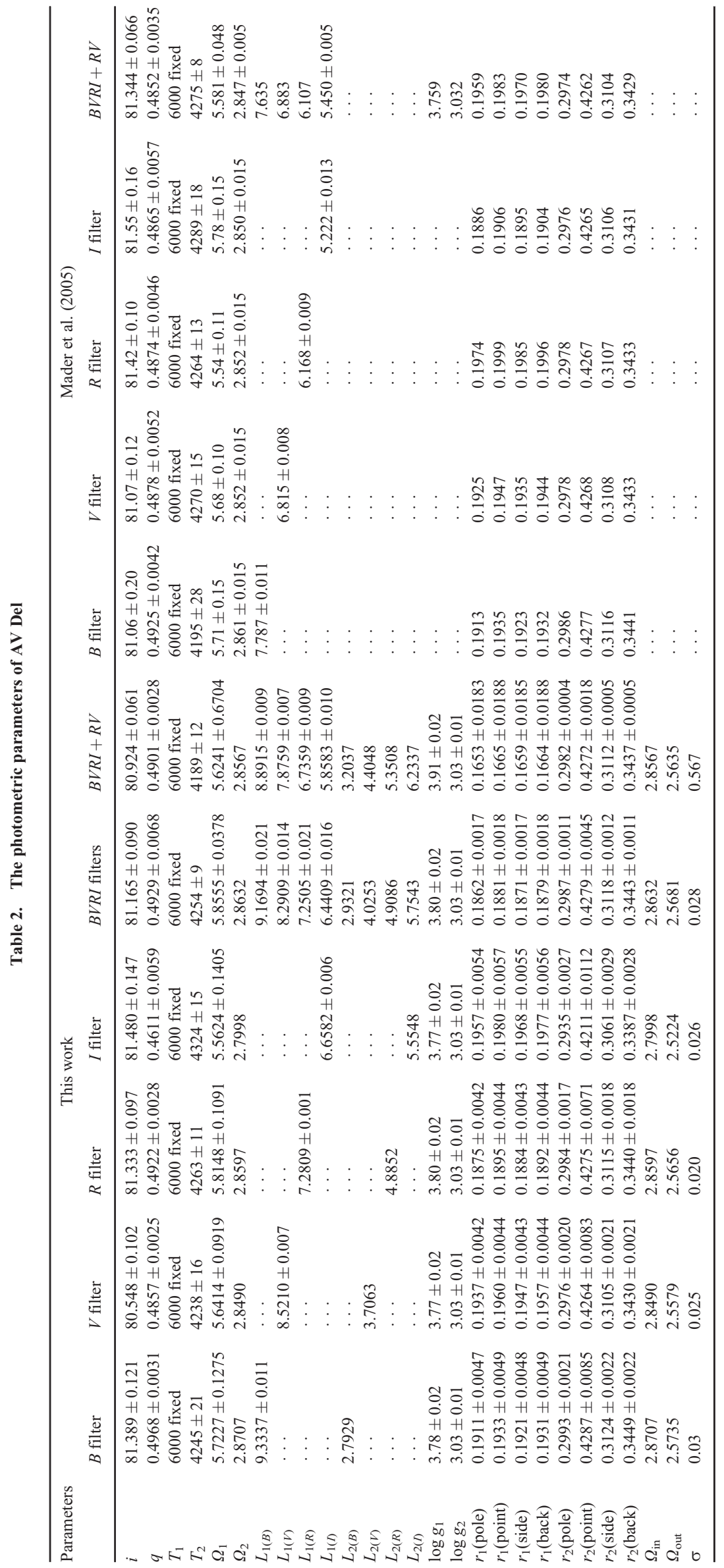



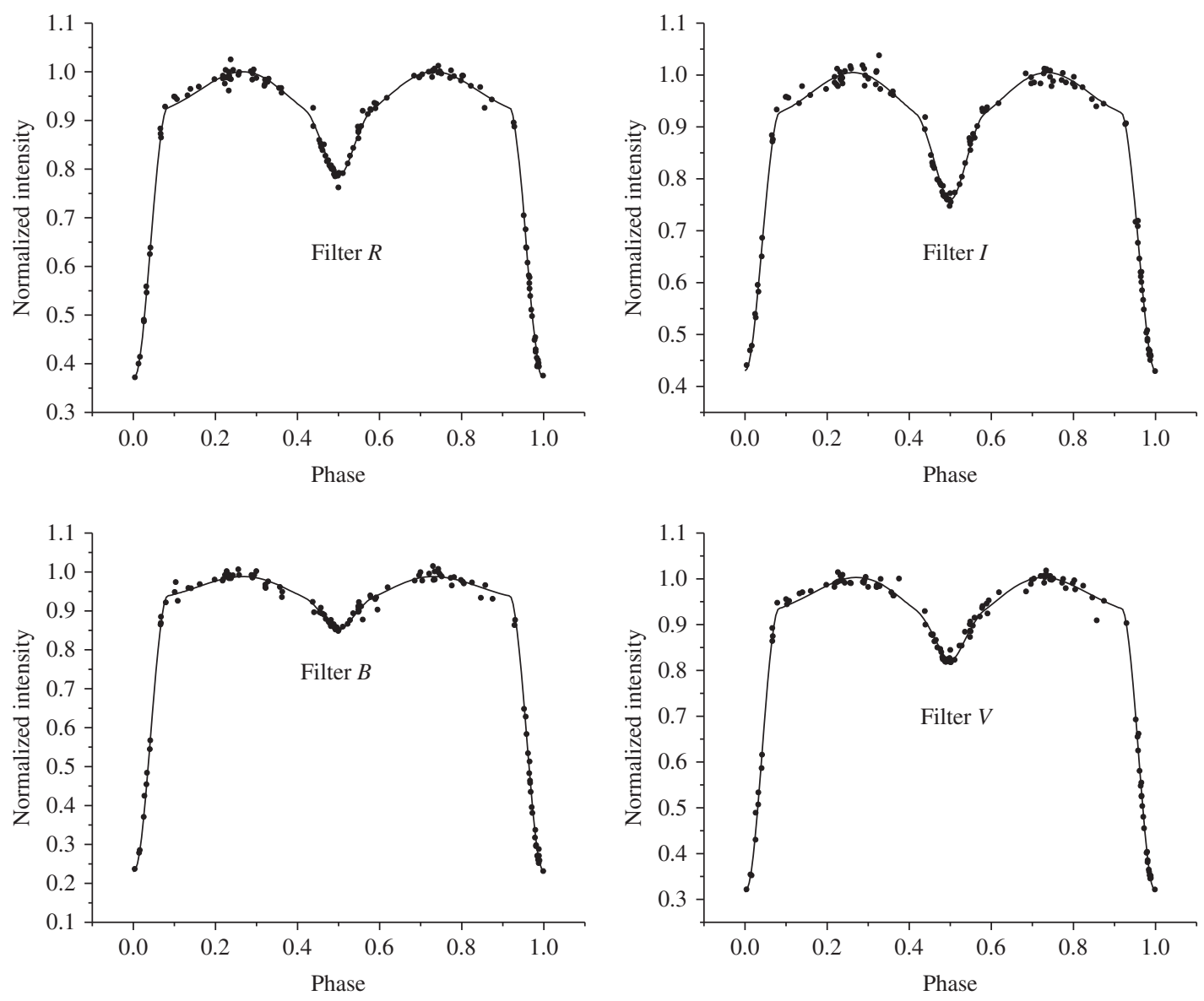

Figure 1 The observed and theoretical light curves of AV Del. The theoretical light curves are drawn on the basis of the values derived via the wD code. Solid circles indicate the observed data and continuous lines show the theoretical light curves. The upper left and right diagrams show the $R$ and $I$ filters, respectively, while the lower left and right diagrams show the $B$ and $V$ filters, respectively.

The temperature of the primary was held fixed at the spectroscopic value of $T_{1}=6000 \mathrm{~K}$. The monochromatic luminosity of the secondary $\left(L_{2}\right)$ was computed by the program directly from the temperatures of the primary and secondary, the luminosity of the primary, the blackbody radiation laws, and the geometry of the system. Other wD code parameters were kept fixed, along with solutions.

Initially, values were obtained in a mode appropriate for semi-detached systems following the expectations based on the characteristics of AV Del. Specifically, mode 5 was used for the secondary which is filling its Roche lobe. The detached configuration was also tried to complete the work. As with Mader et al. (2005), the optimal results were determined with the semi-detached configuration.

The light-curve program (LC) of WD code was implemented to employ an initial set of values for the next round of solutions using the differential corrections main program (DC) of the WD code. After several runs of the DC program, the final set of values were obtained. As mentioned earlier, the assumption $l_{3} \neq 0.0$ never resulted in satisfactory fits to the observations; however, a valid solution was obtained for $l_{3}=0.0$. It can be inferred from this that the best solution is obtained without accounting
Table 3. The spectroscopic parameters of AV Del

\begin{tabular}{lcr}
\hline Parameters & This work & Mader et al. (2005) \\
\hline$V_{\gamma}\left(\mathrm{km} \mathrm{s}^{-1}\right)$ & $-64.141 \pm 0.181$ & $-63.14 \pm 0.24$ \\
$a\left(R_{\odot}\right)$ & $13.3192 \pm 0.0347$ & $13.34 \pm 0.09$ \\
\hline
\end{tabular}

for any additional effects like an accretion disc. The results of photometric solutions with the final elements of the system are given in Table 2. The theoretical light curves computed with the results (continuous line) for each filter are shown in Figure 1.

Using the radial-velocity data from Mader et al. (2005), the orbital elements of the system were obtained. As with the photometric solutions, the wD code was used for the spectroscopic solutions. Values were found for the orbital semi-major axis $(a)$ and the radial velocity of the binary system center of mass $\left(V_{\gamma}\right)$. Also, the ephemeris adopted is the same as the one specified in equation (2). The results of the spectroscopic solutions are given in Table 3. Using the final elements of the objects, the theoretical radial-velocity curves (continuous lines) are shown in Figure 2. Also, all uncertainties given in Tables 2 and 3 are standard errors as reported by the WD code. 
Using the obtained results of the light and radialvelocity curves, the absolute parameters of the system were calculated. The results are listed in Table 4 . The absolute dimensions were determined by using the following formulae:

$$
\begin{gathered}
f_{1}\left(M_{1}, M_{2}, i\right)=\left(M_{2} \sin i\right)^{3} /\left(M_{1}+M_{2}\right)^{2}, \\
L_{1,2} / L_{\odot}=\left(R_{1,2} / R_{\odot}\right)^{2}\left(T_{1,2} / T_{\odot}\right)^{4}, \\
\rho_{1,2} / \rho_{\odot}=\left(0.01344 M_{1,2}\right) /\left[\left(M_{1}+M_{2}\right) P^{2} r_{1,2(\text { side })}^{3}\right],
\end{gathered}
$$

where $f_{1}$ is the mass function and $\rho_{1}$ and $\rho_{2}$ are the mean mass densities of the primary and secondary, respectively.

The potential fillout percentage (fillout) for the components can be calculated from the following formula:

$$
\text { fillout }_{1,2}=\frac{\Omega_{\text {in }}}{\Omega_{1,2}} \times 100 .
$$

where $\Omega_{i n}$ is the critical inner potential and $\Omega_{1}$ and $\Omega_{2}$ are the modified gravitational potentials of the primary and secondary components, respectively.

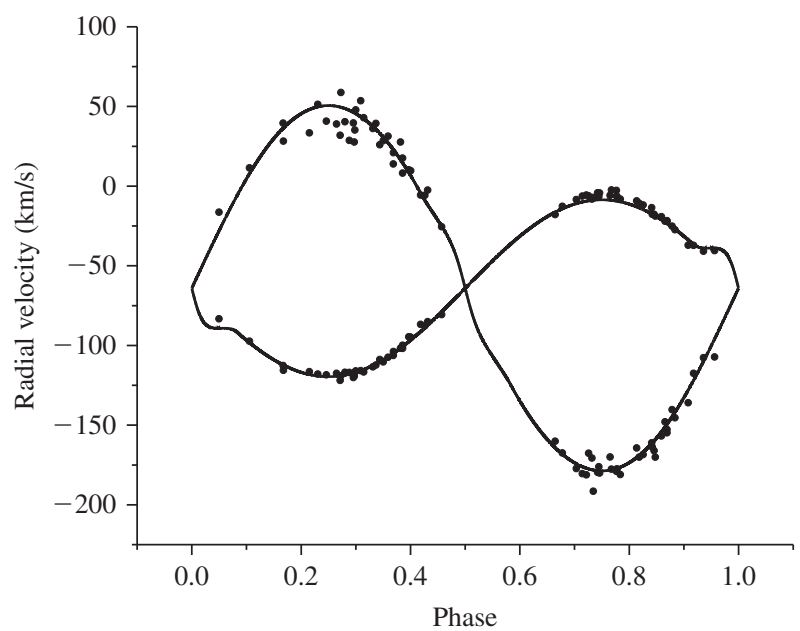

Figure 2 The observed and theoretical radial-velocity curves of AV Del. The theoretical radial-velocity curves are drawn on the basis of the values derived using the WD code. Solid circles show the observed data and continuous lines indicate the theoretical radial-velocity curves.
Also, $K_{1,2}$ and $a_{1,2} \sin i$ were calculated using the following formulae:

$$
\begin{gathered}
K_{1,2}=\frac{2 \pi a_{1,2} \sin i}{P}, \\
a_{1} \sin i=\left[\frac{G P^{2} f_{1}\left(M_{1}, M_{2}, i\right)}{4 \pi^{2}}\right]^{\frac{1}{3}}, \\
a_{2} \sin i=\frac{a_{1} \sin i}{q} .
\end{gathered}
$$

The results are shown in Table 5. On the basis of these values, the configuration of the components was drawn using the BINARY MAKER 2.0 (Bradstreet 1993) software, as shown in Figure 3.

\section{SHELLSPEC Solutions}

As already mentioned, the wD code is not able to study in detail the environmental bodies of binary systems, thus the assumption in accordance with $l_{3} \neq 0.0$ did not result in a good fit to the observed data. Therefore, the SHELLSPEC code was used to solve the light curve of AV Del to determine whether or not the system has an environmental body such as a disc, jet, stream, spot or shell. It is believed that this determination is necessary because it is predicted that Algol-type binaries may contain the objects. Specifically, the secondary star of AV Del fills its Roche lobe, hence the mass transfer probably occurs in the system and forms one or more objects. Besides, the distance between two components is sufficient that one can envisage the shape of the disc or stream.

The SHELLSPEC code is designed to solve simple radiative transfer along the line of sight in three-dimensional moving media. The scattered light from a central object is taken into account assuming an optically thin environment (Budaj \& Richards 2004). Output intensities are then integrated through the two-dimensional projection surface of a three-dimensional object. The assumptions of the code include local thermodynamic equilibrium (LTE) and optical known-state quantities and velocity fields in three dimensions (Budaj \& Richards 2004). Using the results obtained in section 3 , the parameters of a third body were sought in the system using the SHELLSPEC code. In the code, both objects, the primary and the companion (secondary), may be shaped according to the

Table 4. The absolute elements of the binary system AV Del

\begin{tabular}{lcccccc}
\hline Parameters & & \multicolumn{2}{c}{ This work } & & \\
& $B$ filter & $V$ filter & $R$ filter & $I$ filter & $B V R I$ filters & $B V R I+R V$ \\
\hline$M_{1} / M_{\odot}$ & $1.426 \pm 0.025$ & $1.437 \pm 0.027$ & $1.432 \pm 0.023$ & $1.462 \pm 0.025$ & $1.430 \pm 0.028$ & $1.433 \pm 0.031$ \\
$M_{2} / M_{\odot}$ & $0.709 \pm 0.015$ & $0.699 \pm 0.017$ & $0.704 \pm 0.014$ & $0.674 \pm 0.016$ & $0.706 \pm 0.019$ & $0.702 \pm 0.021$ \\
$R_{1} / R_{\odot}$ & $2.56 \pm 0.02$ & $2.59 \pm 0.02$ & $2.51 \pm 0.01$ & $2.62 \pm 0.02$ & $2.49 \pm 0.03$ & $2.21 \pm 0.03$ \\
$R_{2} / R_{\odot}$ & $4.26 \pm 0.02$ & $4.24 \pm 0.02$ & $4.25 \pm 0.01$ & $4.18 \pm 0.02$ & $4.25 \pm 0.03$ & $4.24 \pm 0.03$ \\
$M_{1}(\mathrm{bol})$ & $2.58 \pm 0.04$ & $2.56 \pm 0.04$ & $2.63 \pm 0.02$ & $2.53 \pm 0.03$ & $2.64 \pm 0.06$ & $2.90 \pm 0.07$ \\
$M_{2}(\mathrm{bol})$ & $2.98 \pm 0.04$ & $3.00 \pm 0.03$ & $2.97 \pm 0.01$ & $2.94 \pm 0.03$ & $2.97 \pm 0.05$ & $3.04 \pm 0.07$ \\
\hline
\end{tabular}




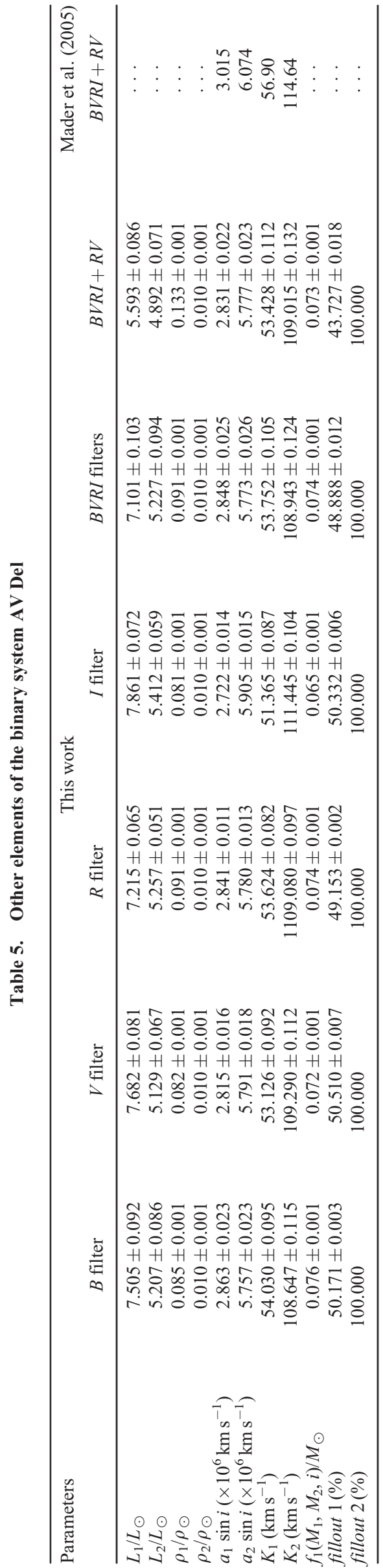

Roche model used for detached, semi-detached or contact systems. As in previous sections, the semi-detached configuration of the Roche model was assumed to apply, and that the primary is a nontransparent uniformly rotating sphere and the secondary component is filling its Roche lobe. The disc surrounds the primary star and eclipses at phase 0.0 . In the model, the eclipse of the secondary star by the disc occurs at phase 0.5 . The disc thickness was assumed to be constant. Also, the black body radiation models were used to find solutions using the SHELLSPEC code.

The limb- and gravity-darkening coefficients used in this code are the same as those described in section 2 , but the reflection effect is not included in these calculations.

Since there were many free parameters in the SHELLSPEC code (because there were several adjustable environmental bodies), at this stage, the parameters of the primary and the secondary (those obtained in section 3 ) were fixed. The effects of the parameters concerning the accretion disc, spot, jet, stream and shell on the light curve were studied to obtain the best fit to the observed data.

Fixing the free parameters of the primary and secondary stars for the values obtained on the basis of the WD code solutions will not lead to invalid results because the WD code is a perfect code for derivation of the parameters of stars in binary systems, and hence we do not centralize our calculations on the free parameters of the primary and secondary and can attend to the other objects. On the other hand, the $R$ filter observations of Mader et al. (2005) were considered for the solutions since, as shown in Table 2 , the accuracy of the results for this filter is greater than for the others.

Solutions were determined for tempsp (effective temperature of the spot), adisc (half the thickness of the disc cylinder), rindc (inner radius of the disc), routdc (outer radius of the disc), tempdc (effective temperature of the disc), densdc (mean density of the disc), anedc (the electron number density of the disc), edendc (the exponent of the power-law behavior of the densities $\rho \sim r^{\eta}$ in the disc) and $v t r b d c$ (microturbulence). The parameters were allowed to vary one by one while the others were kept fixed.

First, the code was run assuming there was no third body, but it failed to obtain a good fit to the observed data. Then a disc alone was included as a third body. On the basis of this configuration, agreement between the observed and the theoretical light curve was very good, although the effect of other bodies (spot, jet, stream, and shell) on the light curve was also examined. Finally, the best fit was obtained when only an accretion disc was considered.

As we see in Figure 4, when the disc is included, the agreement between the observed data and the theoretical light curve is quite good. It should be mentioned that the solution derived in this way is not a unique one. Of course, it shows that the fit is remarkably improved by assuming a disc of certain dimensions and physical parameters. The parameters of the disc are given in Table 6 . The configuration of the system while its primary star is 


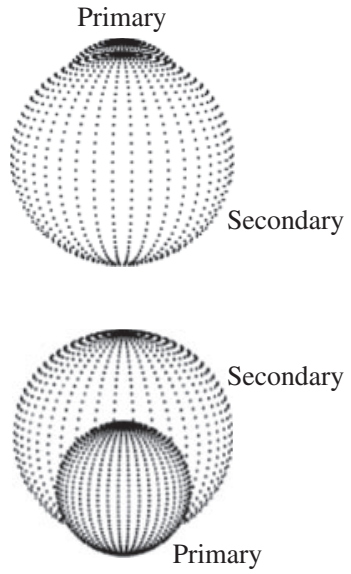

Phase $=0.00$

Secondary
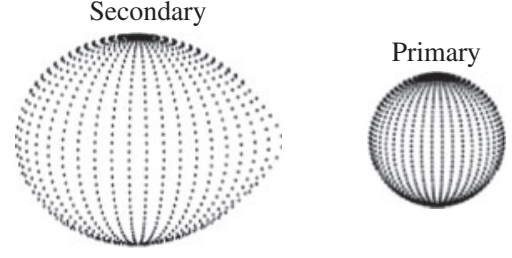

Phase $=0.75$

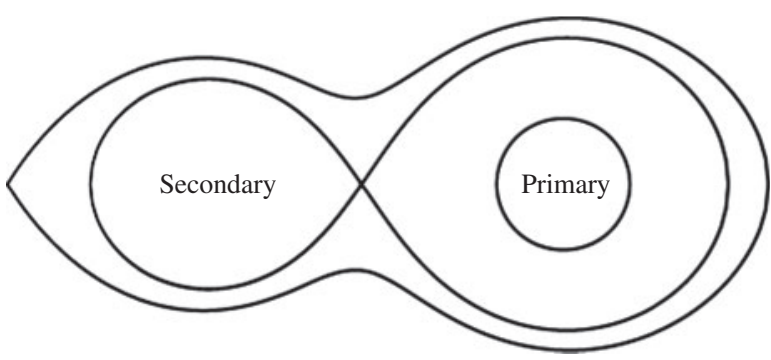

Figure 3 The configuration of the binary system AV Del on the basis of the values derived by using the wD code. It is drawn using the BINARY MAKER 2.0 (Bradstreet 1993) software. From top to bottom, the first, second and third images show the configuration of the system in phases $0.00,0.50$, and 0.75 , respectively. The last image shows the configuration of the system with the Roche lobe.

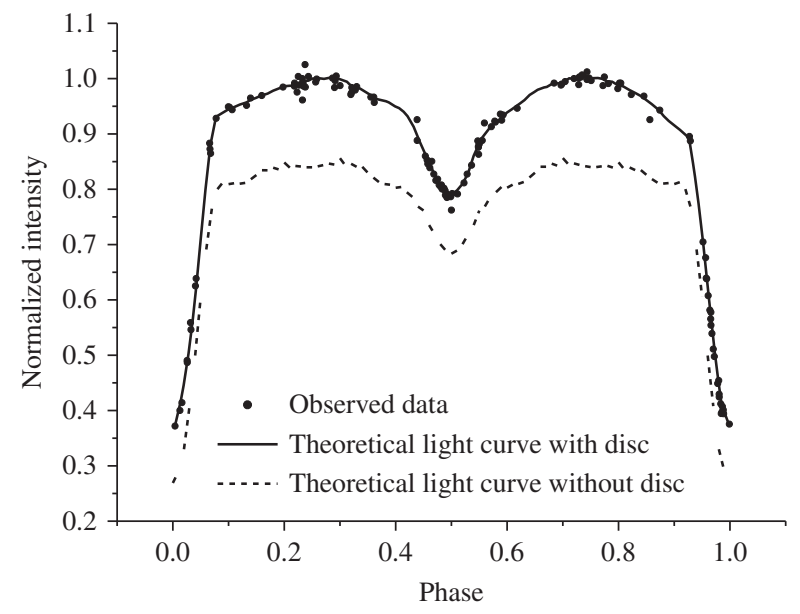

Figure 4 The observed and the theoretical light curves of AV Del. The theoretical light curves are drawn on the basis of the values derived via SHELLSPEC code. The observed data are shown by solid circles and the theoretical light curves are shown by continuous (with disc) and dashed (without disc) lines.
Table 6. The parameters of the accretion disc of AV Del

\begin{tabular}{ll}
\hline Parameters & This work \\
\hline adisc $\left(R_{\odot}\right)$ & 1.2 \\
rindc $\left(R_{\odot}\right)$ & 3.5 \\
routdc $\left(R_{\odot}\right)$ & 8.0 \\
tempdc $(K)$ & 5700 \\
densdc $\left(\mathrm{g} \mathrm{cm}^{-3}\right)$ & $33 \times 10^{-15}$ \\
anedc $\left(\mathrm{cm}^{-3}\right)$ & $21 \times 10^{9}$ \\
edendc & -1 \\
vtrbdc $\left(\mathrm{km} \mathrm{s}^{-1}\right)$ & 90 \\
\hline
\end{tabular}

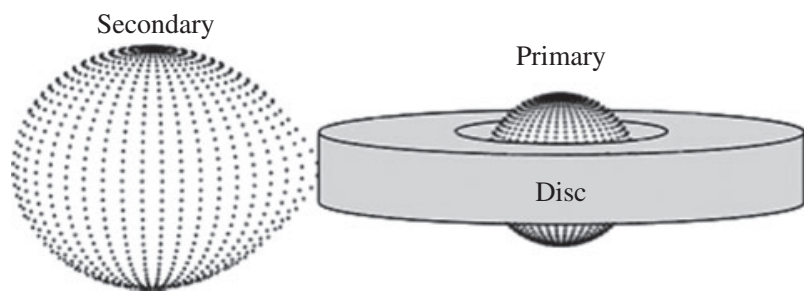

Figure 5 The configuration of the binary system AV Del on the basis of values derived using the SHELLSPEC code. As we see, the primary star is surrounded by an accretion disc.

surrounded by the accretion disc on the basis of the values of Table 6 is shown in Figure 5.

\section{Conclusion}

The latest version of wD code was applied to solve the light and radial-velocity curves of the eclipsing binary system AV Del. The main purpose was to check the possibility of the presence of an accretion disc in the system. However, this tool failed to find any third light in the AV Del system. The wD code was used to determine the absolute parameters of the system. The values obtained are very close to those determined by Mader et al. (2005). The noticeable differences between these values and theirs are seen in the combined light and radialvelocity curves $(B V R I+R V)$ solutions for $i\left(0.42^{\circ}\right)$ and $T_{2}$ $(86 \mathrm{~K})$. On the whole, there is no great difference between the two sets of values, but the present values are more accurate than the former.

The SHELLSPEC code was employed to solve the light curve of AV Del. A new configuration for the system was presented in terms of an accretion disc surrounding the primary star. As shown in Table 6, the accretion disc has the shape of a cylinder. The vertical half-thickness $\left(1.2 R_{\odot}\right)$ is comparable to the radius of the primary star. The distance between the primary and the secondary centers is about $13.3 R_{\odot}$ and the outer radius of the disc is $8 R \odot$. Since the radii of the primary and the secondary are about $2.6 R_{\odot}$ and $4.2 R_{\odot}$, respectively, the outer limb of the disc will be at a distance of about $1.1 R_{\odot}$ from the surface of the secondary. Therefore, the results are realistic. According to the disc dimensions, it is a thick disc in 


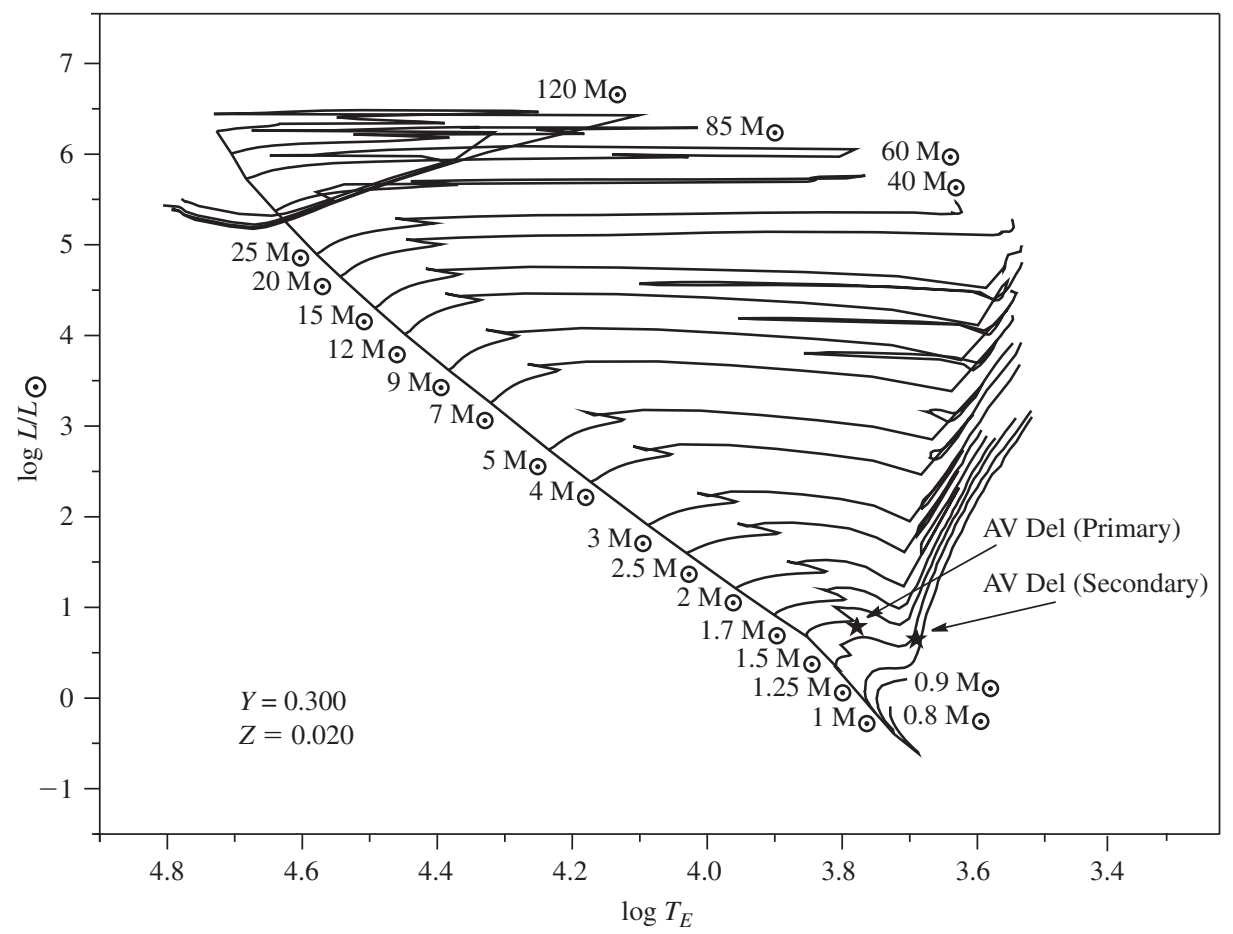

Figure 6 HR diagram for the eclipsing binary system AV Del. The locations of the components are indicated with star symbols. The evolutionary tracks are computed for the exact mass measured for the stars (Schaller et al. 1992) and for a metal abundance of $Z=0.020$.

which the temperature is greater than that of the secondary and slightly less than that of the primary.

Generally, on the basis of the results, AV Del is found to have an accretion disc, while Mader et al. (2005) rejected the existence of activity in the system as they found no obvious signs of activity. However, since there are many free parameters for the disc, it cannot be claimed that the fit is a unique solution (Budaj et al. 2005), but the existence of the disc in the system is certain. Therefore, the period changes seen in the system (Qian, 2002) can be related to the mass transfer between two components which has created the accretion disc.

Finally, Popper (1996) has noted although the two components appear to have comparable luminosities, the hotter and smaller component has the larger mass, with a very provisional mass ratio of about 1.9 . These results are in line with his study.

According to the results, AV Del is a semi-detached system in which the primary and secondary components have filled almost $49 \pm 1$ and 100 percent of their respective critical Roche lobes.

The absolute elements of both components determine the evolutionary state of the system. On the basis of the results, the components of AV Del in the HR diagram were compared with the evolutionary track computed for the exact mass measured for the stars (Schaller et al. 1992) and for a metal abundance of $Z=0.020$ (Figure 6). It appears that AV Del includes a main-sequence primary star, whereas the secondary is on its way to the giant stage.

\section{Acknowledgments}

We wish to thank Dr. Jano Budaj for his very useful comments at different stages of the work and Professor Walter Van Hamme for his very useful help for calculating the error bars of the absolute dimensions. Also we would like to thank the referees for their comments and suggestions.

\section{References}

Bradstreet, D. H., 1993, Light curve modeling of eclipsing binary stars (Springer-Verlag), 151

Budaj, J. \& Richards, M. T., 2004, Contrib. Astron. Obs. Skalanté Pleso, 34, 167

Budaj, J., Richards, M. T. \& Miller, B., 2005, ApJ, 623, 411

Halbedel, E. M., 1984, IBVS, 2549, 1

Haffmeister, C., 1935, Astron. Nature, 255, 401

Klinglesmith, D. A. \& Sobieski, S., 1970, AJ, 75, 175

Lucy, L. B., 1967, Zeit. Fur Astrophysic, 65, 89

Mader, J. A., Torres, G., Marshall, L. A. \& Rizvi, A., 2005, AJ, 130, 234

Popper, D. M., 1996, ApJS, 106, 133

Qian, S., 2002, Ap\&SS, 282, 399

Richards, M. T. \& Albright, G. E., 1999, ApJS, 123, 537

Rucinski, S. M., 2001, AJ, 122, 1007

Schaller, G., Schaerer, D., Meyent, G. \& Maeder, A., 1992, A\&AS, 96, 269

Van Hamme, W., 1993, AJ, 106, 2096

Van Hamme, W. \& Wilson, R. E., 2007, ApJ, 661, 1129

Wilson, R. E., 1979, ApJ, 234, 1054

Wilson, R. E., 1990, ApJ, 356, 613

Wilson, R. E. \& Devinney, E. J., 1971, ApJ, 166, 605

Zucker, S. \& Mazeh, T., 1994, ApJ, 420, 806 